\title{
Facile Approach to Synthesize Gold Nanorod@Polyacrylic Acid/ Calcium Phosphate Yolk-Shell Nanoparticles for Dual-Mode Imaging and pH/NIR-Responsive Drug Delivery
}

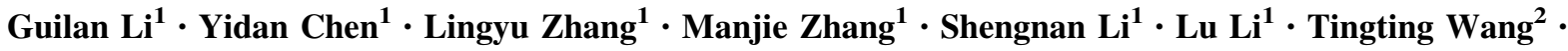 \\ Chungang Wang ${ }^{1}$
}

Received: 30 June 2017/ Accepted: 22 August 2017/Published online: 13 October 2017

(C) The Author(s) 2017. This article is an open access publication

\section{Highlights}

- We report a facile strategy to fabricate gold nanorod@polyacrylic acid/calcium phosphate (AuNR@PAA/CaP) yolkshell nanoparticles.

- The as-obtained AuNR@PAA/CaP yolk-shell nanoparticles (NPs) possess ultrahigh doxorubicin (DOX) loading capability (1 mg DOX/mg NPs), superior photothermal conversion property (26\%) and $\mathrm{pH} /$ near-infrared (NIR) dualresponsive drug delivery performance, which were employed for synergic dual-mode X-ray computed tomography (CT)/photoacoustic (PA) imaging and chemo-photothermal cancer therapy.

- This work brings new insights for the synthesis of multifunctional nanomaterials and extends theranostic applications.

\begin{abstract}
A facile strategy to fabricate gold nanorod@polyacrylic acid/calcium phosphate (AuNR@$\mathrm{PAA} / \mathrm{CaP}$ ) yolk-shell nanoparticles (NPs) composed with a $\mathrm{PAA} / \mathrm{CaP}$ shell and an AuNR yolk is reported. The asobtained AuNR@PAA/CaP yolk-shell NPs possess ultrahigh doxorubicin (DOX) loading capability (1 mg DOX/ mg NPs), superior photothermal conversion property (26\%) and $\mathrm{pH} /$ near-infrared (NIR) dual-responsive drug delivery performance. The released DOX continuously increased due to the damage of the $\mathrm{CaP}$ shell at low $\mathrm{pH}$ values. When the DOX-loaded AuNR@PAA/CaP yolk-shell NPs were
\end{abstract}

Electronic supplementary material The online version of this article (doi:10.1007/s40820-017-0155-3) contains supplementary material, which is available to authorized users.

Tingting Wang

wangtt@cust.edu.cn

$\triangle$ Chungang Wang

wangcg925@nenu.edu.cn

1 Faculty of Chemistry, Northeast Normal University, Changchun 130024, People's Republic of China

2 School of Chemistry and Environmental Engineering, Changchun University of Science and Technology, Changchun 130022, People's Republic of China exposed to NIR irradiation, a burst-like drug release occurs owing to the heat produced by the AuNRs. Furthermore, AuNR@PAA/CaP yolk-shell NPs are successfully employed for synergic dual-mode X-ray computed tomography/photoacoustic imaging and chemo-photothermal cancer therapy. Therefore, this work brings new insights for the synthesis of multifunctional nanomaterials and extends theranostic applications.

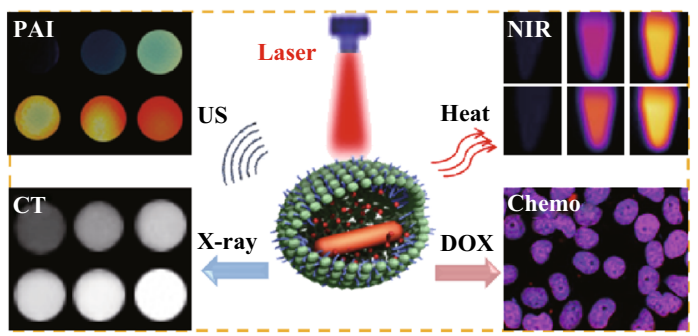

Keywords Yolk-shell structure - Calcium phosphate . Dual-mode imaging · Photothermal therapy · Drug delivery 


\section{Introduction}

Multifunctional nanoparticles (NPs) with complementary capacities of multimodal imaging and therapeutic functions have drawn extensive attention in biomedical areas [1-6]. Nowadays, calcium phosphate (CaP) NPs have gained increasing attention in anticancer drug delivery because of their excellent biocompatibility and $\mathrm{pH}$-sensitivity, originating from their chemical nature and mimics the inorganic component of biological hard tissues, such as bone and tooth [7-11]. However, owing to the lack of the theranostic capability, single CaP NPs are difficult to achieve simultaneous imaging and cancer theranostics. The main strategy turns to synthesize CaP-based multifunctional NPs with the capability of diagnosis and therapeutics. For example, Liu et al. developed a synthetic route to obtain amphiphilic gelatin-iron oxide core/CaP shell NPs, integrating magnetic resonance imaging and chemotherapy for killing cancer cells [12]. It should be noted that sole modality imaging or therapy cannot enhance the anticancer efficiency in comparison with multimodal imaging and multiple therapeutic. Therefore, it is absolutely imperative to propose a simple synthetic method for fabricating CaPbased multifunctional NPs that possess the capacities of simultaneous dual-mode imaging diagnosis and chemophotothermal therapy.

Among numerous photothermal nanomaterials, the optical property of gold nanorod (AuNRs) presented good photothermal therapy (PTT) effect owing to their tunable localized surface plasmon resonance (LSPR) across the NIR region [13-27]. Meanwhile, AuNRs possess photoacoustic (PA) and X-ray computed tomography (CT) imaging capacity, due to the strong near-infrared (NIR) absorption and X-ray opacity [28, 29]. Recently, Lu and co-workers prepared yolk-shell AuNR@hollow periodic mesoporous organosilica nanospheres only for chemo-photothermal therapy of breast cancer [30]. However, their materials still have problems that need to be tackled including large particle size, poor biocompatibility and low drug loading capability. Moreover, up to now, there have been no reports on the synthesis of CaP-based yolk-shell NPs composed of a CaP shell and a removable AuNR yolk. It is worth mentioning that the yolk-shell architecture is a promising candidate for developing drug loading systems compared with the core-shell structure, owing to the unique cavity, large surface area and excellent loading capacity [31-41]. Hence, developing a facile synthetic strategy to fabricate welldispersed AuNR core/CaP shell NPs with ultrahigh doxorubicin (DOX) loading capability and pH/NIR dual-responsive drug delivery performance for dual-mode CT/PA imaging and synergic chemo-photothermal therapy of cancer cells still remains a great challenge.
Herein, we have successfully developed for the first time a mild and facile route to fabricate AuNR@PAA/CaP yolk-shell NPs for synergistic dual-mode CT/PA imaging and chemo-photothermal therapy of cancer cells.

\section{Experimental Details}

\subsection{Materials}

Hydrogen tetrachloroaurate trihydrate $\left(\mathrm{HAuCl}_{4} \cdot 3 \mathrm{H}_{2} \mathrm{O}\right)$, silver nitrate $\left(\mathrm{AgNO}_{3}\right)$, hexadecyltrimethylammonium bromide (CTAB), L-ascorbic acid (LAA), sodium borohydride $\left(\mathrm{NaBH}_{4}\right)$, polyacrylic acid $\left(\mathrm{PAA}, M_{\mathrm{W}} \approx 1800\right)$ and doxorubicin hydrochloride (DOX) were purchased from Sigma-Aldrich (USA). Disodium hydrogen phosphate $\left(\mathrm{Na}_{2} \mathrm{HPO}_{4}\right)$, hydrochloric acid $(\mathrm{HCl}, 37 \%$ weight in water), tetraethylorthosilicate (TEOS), methanol (HCHO, 37\% weight in water), anhydrous ethanol $\left(\mathrm{CH}_{3} \mathrm{CH}_{2} \mathrm{OH}\right)$, sodium hydroxide $(\mathrm{NaOH})$, calcium hydroxide $\left(\mathrm{Ca}(\mathrm{OH})_{2}\right)$ and isopropyl alcohol (IPA) were purchased from Sinopharm Chemical Reagent Beijing Co. Ltd. All chemicals were used without any further purification. All glassware was first cleaned with freshly prepared aqua regia and extensively rinsed with water before it was used. The deionized (DI) water was used in all experiments.

\subsection{Characterization}

Transmission electron microscope (TEM) measurements were taken on a JEOL-2100F transmission electron microscope at $200 \mathrm{kV}$ (Hitachi, Japan). The irradiation was performed using a NIR laser with a center wavelength of 808 nm (Beijing Kaipulin Optoelectronic Technology Co.). The scanning electron microscopy (SEM) and energy dispersive X-ray (EDX) spectrum were carried out with a JEOL JSM-7610F scanning electron microscope. X-ray photoelectron spectra (XPS) were measured on an ECSALAB 250 using non-mono-chromatized Al- $K \alpha$ radiation. Fourier transform infrared (FTIR) spectra were performed by a Magna 560 FTIR spectrometer (Nicolet, USA). The $\mathrm{UV}-\mathrm{Vis}$ spectra were recorded at room temperature on a Japan JASCO V-570 spectrometer fluorescence spectrophotometer. The inductively coupled plasma atomic emission spectroscopy (ICP-AES) was determined by a Leeman ICP-AES Prodigy instrument.

\subsection{Synthesis of AuNRs}

AuNRs with an aspect ratio of $\sim 4$ was prepared by a seedmediated growth method using CTAB surfactants as reported previously [42]. Firstly, the synthesis of gold nanoseeds: $\mathrm{HAuCl}_{4}(10 \mathrm{mM}, 0.25 \mathrm{~mL})$ and CTAB $(0.1 \mathrm{M}$, 
$10 \mathrm{~mL}$ ) were added into a $10 \mathrm{~mL}$ glass bottle with gentle mixing before a freshly prepared and ice-bathed $\mathrm{NaBH}_{4}$ solution $(10 \mathrm{mM}, 0.6 \mathrm{~mL})$ was injected and then the mixture was magnetically stirred until the color changed from golden yellow to brown indicating that $3.5 \mathrm{~nm}$ gold nanoseeds were obtained. The seed solution was then kept at $30{ }^{\circ} \mathrm{C}$ for at least $2 \mathrm{~h}$ before usage. Secondly, the growth of gold nanorods: $\mathrm{AgNO}_{3}(10 \mathrm{mM}, 1 \mathrm{~mL})$ and $\mathrm{HAuCl}_{4}$ $(10 \mathrm{mM}, 5 \mathrm{~mL})$ were added into CTAB $(0.1 \mathrm{M}, 100 \mathrm{~mL})$ in a $250 \mathrm{~mL}$ glass bottle, followed by the addition of $\mathrm{HCl}$ $(0.1 \mathrm{M}, 700 \mu \mathrm{L})$, LAA $(0.1 \mathrm{M}, 700 \mu \mathrm{L})$ and gold seeds $(100 \mu \mathrm{L})$. The growth solution was left at $30{ }^{\circ} \mathrm{C}$ overnight. Finally, CTAB-stabilized AuNRs were prepared, then the sample solution was centrifuged several times to remove superfluous CTAB and redispersed into $100 \mathrm{~mL}$ of DI water for further use.

\subsection{Synthesis of AuNR@ $\mathrm{mSiO}_{2}$ Core-Shell NPs}

When the $\mathrm{pH}$ value of $16 \mathrm{~mL}$ AuNRs solution was adjusted to about 10 with $\mathrm{NaOH}(0.1 \mathrm{M})$ solution under stirring, $30 \mu \mathrm{L}$ of TEOS was subsequently injected slowly under vigorous stirring. The reaction mixture was allowed to proceed for $4 \mathrm{~h}$ to form an approximately 15-nm thick silica layer on the surface of AuNRs. Finally the AuNR@ $\mathrm{mSiO}_{2}$ core-shell NPs were isolated by centrifugation and washed with DI water several times and then redissolved in $5 \mathrm{~mL}$ DI water for further use.

\subsection{Synthesis of AuNR@PAA/CaP Yolk-Shell NPs}

In a $50 \mathrm{~mL}$ of flask, $8 \mathrm{mg} \mathrm{Ca}(\mathrm{OH})_{2}$ and $100 \mu \mathrm{L}$ of PAA aqueous solution $\left(0.2 \mathrm{~g} \mathrm{~mL}^{-1}\right)$ were firstly added to $10 \mathrm{~mL}$ DI water under magnetic stirring. In succession, $5 \mathrm{~mL}$ of AuNR@ $\mathrm{mSiO}_{2}$ core-shell NPs solution was dispersed into the solution to form a suspension. Then, $20 \mathrm{~mL}$ of IPA was dripped into the suspension under magnetic stirring. Afterward, $24 \mathrm{mg} \mathrm{Na}{ }_{2} \mathrm{HPO}_{4}$ was added to the above mixed suspension under magnetic stirring for $10 \mathrm{~h}$. After being centrifuged and washed with DI water, the AuNR@mSiO $\mathrm{m}_{2} @ \mathrm{PAA} / \mathrm{CaP}$ core-shell NPs were obtained. Finally, after etching the $\mathrm{mSiO}_{2}$ layer, the AuNR@PAA/ CaP yolk-shell NPs were obtained.

\subsection{DOX Loading and Release}

The amount of DOX loaded into the AuNR@PAA/CaP yolk-shell NPs was measured by UV-Vis spectrophotometer. After mixing $\operatorname{DOX}\left(0.1 \mathrm{~mL}, 10 \mathrm{mg} \mathrm{mL}^{-1}\right)$ with AuNR@PAA/CaP yolk-shell NPs $(0.8 \mathrm{~mL}$, $1.2 \mathrm{mg} \mathrm{mL}^{-1}$ ), and shaking overnight, DOX-loaded AuNR@PAA/CaP yolk-shell NPs were obtained by centrifugation and washed three times with DI water to remove the DOX adsorbed on the surface. Free DOX in the supernatant was determined by measuring the absorbance at $480 \mathrm{~nm}$ in a UV-Vis spectrophotometer. The DOXloading efficiency (LE) was calculated by Eq. 1:

$$
\begin{aligned}
\mathrm{LE}(\%)= & \frac{W_{\text {initial DOX }}-W_{\text {remanent DOX }}}{W_{\text {AuNR@PAA } / \mathrm{CaP}}} \\
& \times 100 \%\left(\mathrm{mg} \mathrm{mg}^{-1}\right)
\end{aligned}
$$

Two portions of the prepared DOX-loaded AuNR@PAA/CaP yolk-shell NPs at equal amount were redispersed in $\mathrm{pH} 7.4$ and $\mathrm{pH} 5.0 \mathrm{PBS}(0.5 \mathrm{~mL})$ and then transferred into pretreated semipermeable dialysis bags at $37^{\circ} \mathrm{C}$, respectively. After the two bags were immersed into $5 \mathrm{~mL}$ of PBS buffer (pH 7.4 and $\mathrm{pH} 5.0$ ) at $37{ }^{\circ} \mathrm{C}$, the amount of released DOX moving into the solution was determined by measuring the absorbance at $480 \mathrm{~nm}$ in a UV-Vis spectrophotometer at selected time intervals. To confirm that the laser irradiation can induce the drug release, another experiment was also carried out under the same procedures. The sample was immersed in PBS buffer at $\mathrm{pH} 5.0$ with NIR irradiation $\left(808 \mathrm{~nm}, 1.0 \mathrm{~W} \mathrm{~cm}^{-2}\right)$ at selected time intervals. DOX concentration in the supernatant was determined by UV-Vis spectrophotometer as well. The samples $(1 \mathrm{mg})$ were put into $\mathrm{pH} 5.0 \mathrm{PBS}(3 \mathrm{~mL})$. The supernatants were collected by centrifugation at selected time intervals and analyzed by ICP-AES to measure the $\mathrm{Ca}$ content.

\subsection{Cell Culture}

Human hepatocellular carcinoma (HeLa) cells were grown as a monolayer in a humidified incubator at $37{ }^{\circ} \mathrm{C}$ in a $95 \%$ air $5 \% \mathrm{CO}_{2}$ in Dulbecco's modified eagle medium (DMEM) supplemented with $10 \%$ fetal bovine serum.

\subsection{The Photothermal Therapy of AuNR@PAA/ CaP Yolk-Shell NPs}

The photothermal effect of AuNR@PAA/CaP yolk-shell NPs was measured in aqueous solution. Briefly, AuNR@ PAA/CaP yolk-shell NPs (1 mL) with various concentrations were exposed to the 808-nm NIR laser (power density $1.0 \mathrm{~W} \mathrm{~cm}^{-2}$ ) for $5 \mathrm{~min}$. The temperature was recorded every $30 \mathrm{~s}$. Subsequently, the photothermal effect in the cell level was analyzed by using calcein AM staining method. Calcein AM can only penetrate in live cells and emit green fluorescence. The cells were seeded in a 24-well plate $\left(2.5 \times 10^{4}\right.$ cells per well $)$ for $24 \mathrm{~h}$. Then, the cells were divided into four groups: group 1 with PBS only; group 2 incubated with AuNR@PAA/CaP yolk-shell NPs (25 $\mu \mathrm{g} \mathrm{mL}^{-1}$ ); group 3 incubated with NIR; group 4 incubated with both NPs and NIR laser. The NIR laser used in this experiment was $1.0 \mathrm{~W} \mathrm{~cm}^{-2}$ and the irradiation 
time was $5 \mathrm{~min}$. After all the treatment, the cells were finally stained with calcein AM.

\subsection{Calculation of the Photothermal Conversion Efficiency $(\boldsymbol{\eta})$}

To evaluate the photothermal conversion efficiency $(\eta)$, the time-dependent temperature increment of the aqueous dispersion $(0.5 \mathrm{mM})$ was recorded under the continuous 808-nm NIR laser irradiation with a power density of $1.0 \mathrm{~W} \mathrm{~cm}^{-2}$. Subsequently, the irradiation source was shut off, and the temperature decrease of the aqueous dispersion was monitored to determine the rate of heat transfer from the dispersion system to the environment. The photothermal conversion efficiency $(\eta)$ of AuNR@PAA/CaP yolkshell NPs was calculated using Eq. 2:

$\eta=\frac{h S\left(T_{\max }-T_{\text {surr }}\right)-Q_{\mathrm{dis}}}{I\left(1-10^{-A_{808}}\right)}$

where $h$ is the heat transfer coefficient, $S$ is the surface area of the container, $T_{\max }$ and $T_{\text {surr }}$ are the equilibrium temperature and ambient temperature, respectively. $Q_{\text {dis }}$ represents the heat dissipation from the light absorbed by container, $I$ is the incident laser power, and $A_{808}$ is the absorption intensity of AuNR@PAA/CaP yolk-shell NPs at $808 \mathrm{~nm}$. The value of $h S$ is derived according to Eq. 3:

$\tau_{s}=\frac{m_{\mathrm{D}} C_{\mathrm{D}}}{h s}$

where $\tau_{\mathrm{s}}$ is the sample system time constant, $m_{\mathrm{D}}$ and $C_{\mathrm{D}}$ are the mass and heat capacity of DI water used as the solvent, respectively. The $Q_{\text {dis }}$ was measured independently using sample cell containing pure water without AuNR@PAA/ CaP yolk-shell NPs.

\subsection{In Vitro Cytotoxicity Evaluation Against HeLa Cells}

The cytotoxicity of empty AuNR@PAA/CaP yolk-shell NPs, free DOX and DOX-loaded AuNR @PAA/CaP yolkshell NPs were evaluated by standard 3-(4,5-dimethylthialzol-2-yl)-2,5-diphenyltetrazolium bromide (MTT) assays. HeLa cells were seeded in a 96-well plate at a density of $2.5 \times 10^{4}$ per well and incubated in the atmosphere of $95 \%$ air and $5 \% \mathrm{CO}_{2}$ at $37{ }^{\circ} \mathrm{C}$ with $10 \%$ fetal bovine serum for $24 \mathrm{~h}$. Then various concentrations of empty AuNR@PAA/CaP yolk-shell NPs, free DOX and DOX-loaded AuNR@PAA/CaP yolk-shell NPs were added, respectively. In another plate, the suspensions of AuNR@PAA/CaP yolk-shell NPs with different concentrations were added into HeLa cells with the 808-nm NIR laser irradiation for $5 \mathrm{~min}$. One row of a 96-well plate was added culture medium only to be a blank control. After incubated for $24 \mathrm{~h}, 20 \mu \mathrm{L}$ of $5 \mathrm{mg} \mathrm{mL}^{-1}$ MTT solution was added to each well for $4 \mathrm{~h}$ incubation. Then, the medium was replaced with DMSO $(150 \mu \mathrm{L})$ to dissolve the MTT formazan crystals. The cell viability can be calculated by using Eq. 4:

Cell viability $(\%)=\frac{A_{\text {(test cells) }}}{A_{\text {(control cells })}} \times 100 \%$

\subsection{CT Imaging of AuNR@PAA/CaP Yolk-Shell NPs In Vitro}

AuNR@PAA/CaP yolk-shell NPs with the Au concentrations in a range of $0-0.25 \mathrm{M}$ were poured into tubes and placed in a self-made scanning holder. CT images were acquired under the following parameters: thickness, $1.0 \mathrm{~mm}$; pitch, $120 \mathrm{kV}, 280 \mathrm{~mA}$; field of view, $300 \mathrm{~mm}$; gantry rotation time, $4.95 \mathrm{~s}$. CT data were analyzed by recording the Hounsfield units (HUs) for regions of interest. The raw data were reconstructed using 3D-Med software to acquire the $\mathrm{CT}$ images and calculate the $\mathrm{CT}$ values.

\subsection{PA Signal Measurement In Vitro}

To test the linearity of the PA signal as a function of AuNR@PAA/CaP yolk-shell NPs, $0.2 \mathrm{~mL}$ of the AuNR@PAA/CaP yolk-shell NPs aqueous suspension with different $\mathrm{Au}$ concentrations $(0,0.4,0.5,0.7$ and $0.9 \mathrm{mM}$ ) was added to the agar-phantom container and placed in the MOST inVision 128 (iThera) system for signal detection. A complete PA image of the phantom was collected at $808 \mathrm{~nm}$.

\section{Results and Discussion}

\subsection{Synthesis and Characterization of AuNR@PAA/ CaP Yolk-Shell NPs}

As illustrated in Scheme 1, AuNR@mesoporous silica (AuNR@ $\mathrm{mSiO}_{2}$ ) core-shell NPs were formed upon the addition of TEOS into the prepared AuNR solution. The asfabricated AuNR@ $\mathrm{mSiO}_{2}$ core-shell NPs mixed with the PAA-Ca aqueous solution, which obtained by adding $\mathrm{Ca}(\mathrm{OH})_{2}$ powder into PAA aqueous solution. Upon the addition of IPA, the PAA-Ca self-assembled around the surface of AuNR@ $\mathrm{mSiO}_{2}$ core-shell NPs to obtain AuNR@ $\mathrm{mSiO}_{2} @ \mathrm{PAA}-\mathrm{Ca}$ core-shell NPs. Then, AuNR@mSiO $\mathrm{m}_{2} @ \mathrm{PAA} / \mathrm{CaP}$ core-shell NPs were fabricated through the assistance of $\mathrm{Na}_{2} \mathrm{HPO}_{4}$ as the phosphate anion source. Finally, the $\mathrm{mSiO}_{2}$ layer was etched by sodium hydroxide from the core-shell AuNR@mSiO $@ \mathrm{PAA} / \mathrm{CaP}$ NPs to get the yolk-shell AuNR@PAA/CaP NPs. After loading the anticancer drug, DOX, the DOX-loaded yolk- 


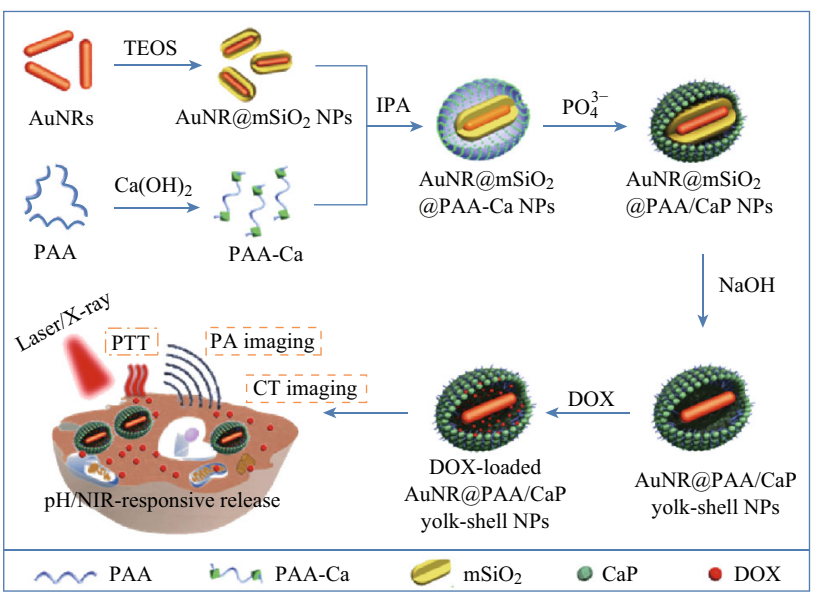

Scheme 1 Schematic illustration of the synthetic strategy for the AuNR@PAA/CaP yolk-shell NPs as pH/NIR-responsive drug carriers for simultaneous dual-mode CT/PA imaging and chemo-photothermal therapy in vitro

shell NPs were utilized as $\mathrm{pH} / \mathrm{NIR}$-responsive drug vehicles for dual-mode CT/PA imaging and chemo-photothermal cancer therapy in vitro.

The TEM image in Fig. 1a shows that the average length and width of AuNRs are around 60 and $15 \mathrm{~nm}$, respectively (about 4:1 aspect ratio). The absorption wavelength of AuNRs can be tuned by changing the AuNR aspect ratio [43]. When the aspect ratio is around 4, a strong LSPR band was observed at around $800 \mathrm{~nm}$, matching the $808-\mathrm{nm}$ NIR laser irradiation well, thereby ensuring effective heat production. With the addition of TEOS and ammonium hydroxide into the as-prepared AuNR solution, the AuNR@ $\mathrm{mSiO}_{2}$ coreshell NPs are about $80 \mathrm{~nm}$ in length and $45 \mathrm{~nm}$ in width (Fig. 1b). To synthesize monodispersed AuNR@ $\mathrm{mSiO}_{2} @$ PAA-Ca core-shell NPs, the PAA-Ca aqueous solution was firstly obtained by adding $\mathrm{Ca}(\mathrm{OH})_{2}$ powder into PAA aqueous solution due to the acid-base neutralization reaction [2]. Then, the as-fabricated AuNR@ $\mathrm{mSiO}_{2}$ core-shell NPs and IPA were added to the PAA-Ca aqueous solution in sequence under vigorous stirring to form AuNR@mSiO $\mathrm{m}_{2} @$ PAA-Ca core-shell NPs with a PAA-Ca shell about $10 \mathrm{~nm}$ (Fig. 1c), due to the insolubility of PAA$\mathrm{Ca}$ in the presence of IPA. The subsequent addition of $\mathrm{Na}_{2-}$ $\mathrm{HPO}_{4}$ powder as the phosphate anion source induced the formation of a PAA-CaP shell, the finally gained well-dispersed AuNR@mSiO $\mathrm{m}_{2} @ \mathrm{PAA} / \mathrm{CaP}$ core-shell NPs with the average length and width around 100 and $65 \mathrm{~nm}$, respectively (Fig. 1d). Then, the resulting AuNR@ $\mathrm{mSiO}_{2} @ \mathrm{PAA} /$ $\mathrm{CaP}$ core-shell NPs were treated in diluted base solution for $1 \mathrm{~h}$ to generate AuNR@PAA/CaP yolk-shell NPs with a thin PAA/CaP shell of approximately $10 \mathrm{~nm}$ in thickness (Fig. 1e), which is shown in Fig. 1c. The oval-shaped cavity formation with the lower contrast in the TEM image made the hollow structure pronounced and proved the synthesis of yolk-shell NPs. The SEM image of the AuNR@PAA/CaP yolk-shell NPs presents a fairly rough surface, which proved the shell is composed of a large amount of small $\mathrm{CaP}$. The formation process of the AuNR@PAA/CaP yolk-shell NPs was monitored by the UV-Vis absorbance spectra in Fig. 1f. The absorption peak around $510 \mathrm{~nm}$ is ascribed to the transverse surface plasmon band of the AuNRs, and the
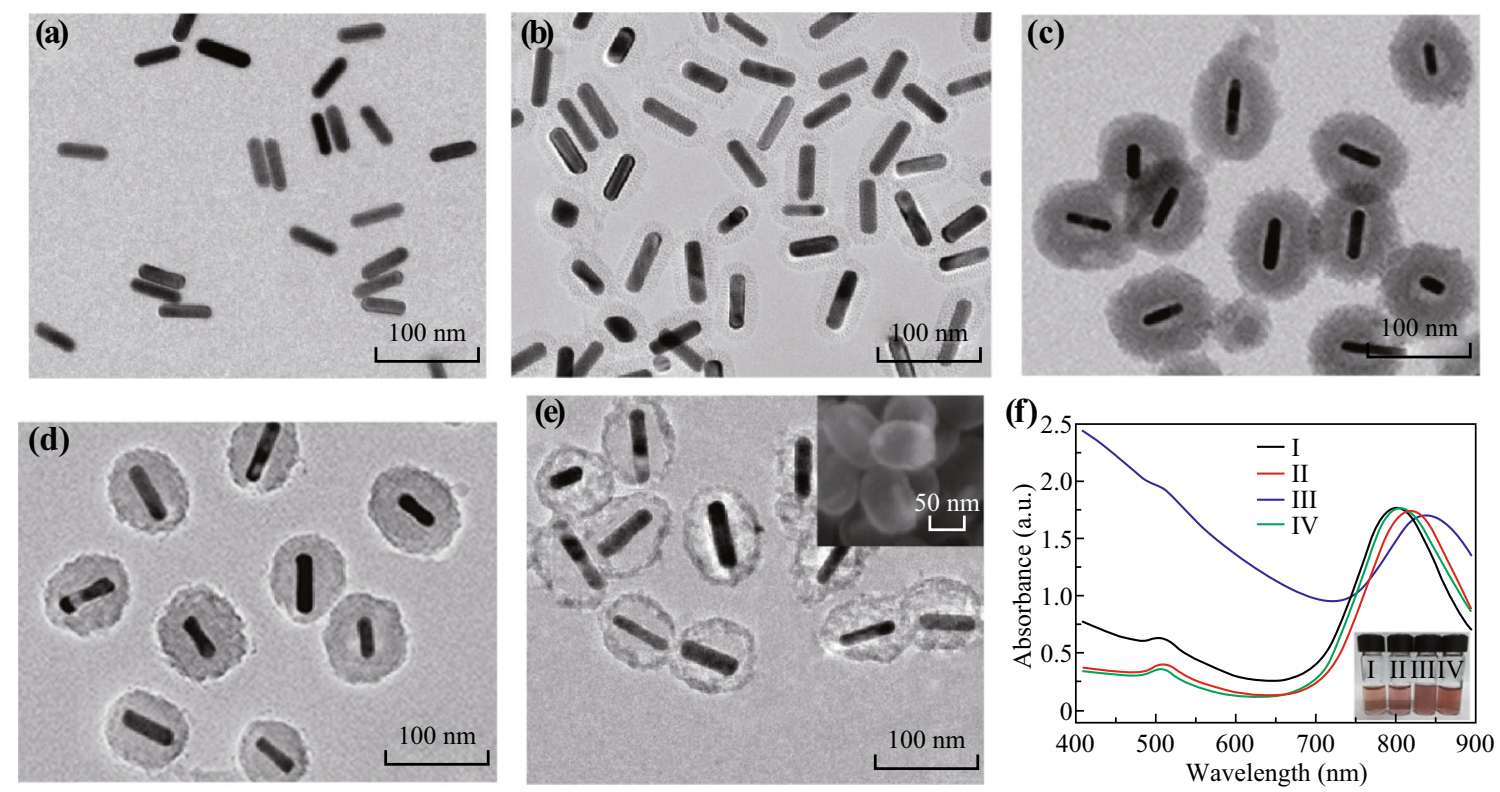

Fig. 1 TEM images of a AuNRs, b AuNR@mSiO ${ }_{2}$ core-shell NPs, c AuNR@mSiO $@$ @PA-Ca core-shell NPs, d AuNR@mSiO $@$ PAA/CaP core-shell NPs, e AuNR@PAA/CaP yolk-shell NPs, the inset shows the SEM image of the yolk-shell NPs, f UV-Vis absorbance spectra of (I) AuNRs, (II) AuNR@mSiO ${ }_{2}$ core-shell NPs, (III) AuNR@mSiO ${ }_{2} @ P A A / C a P$ core-shell NPs and (IV) AuNR@PAA/CaP yolk-shell NPs 
absorption peak around $802 \mathrm{~nm}$ is attributed to the LSPR band. Compared to the absorption spectrum of AuNRs, the LSPR bands of AuNR@mSiO ${ }_{2} \mathrm{NPs}_{\text {and AuNR@mSiO }} @$ PAA/CaP NPs redshift about $18 \mathrm{~nm}$ (from 802 to $820 \mathrm{~nm}$ ) and $38 \mathrm{~nm}$ (from 802 to $840 \mathrm{~nm}$ ), respectively, owing to the difference of the local dielectric function of surrounding medium. While, after dissolving $\mathrm{mSiO}_{2}$ layer, a strong LSPR band of AuNR@PAA/CaP yolk-shell NPs at 806 nm was observed. The above results further verify the successful preparation of AuNR@PAA/CaP yolk-shell NPs. In addition, no obvious aggregation was observed when the AuNR@PAA/CaP yolk-shell NPs were dispersed into water, PBS buffer and Dulbecco's modified eagle medium (DMEM) for $4 \mathrm{~h}$, respectively, reflecting their good stability (Fig. S1, ESI).

To further confirm the details of the AuNR@PAA/CaP yolk-shell NPs, a series of characterization were performed. Figure S2 displays the FTIR spectrum of the AuNR@PAA/CaP yolk-shell NPs. The broad peak around $3500 \mathrm{~cm}^{-1}$ originates from moisture in the samples. The absorption bands at 1558 and $1419 \mathrm{~cm}^{-1}$ are attributed to the characteristic peaks of the carbonyl $(\mathrm{C}=\mathrm{O})$ of the carboxylic acid (-COO-) group, confirming the presence of PAA. Moreover, the characteristic peaks appeared at 565 and $1087 \mathrm{~cm}^{-1}$ can be assigned to the characteristic peaks of $\mathrm{O}-\mathrm{P}-\mathrm{O}$ bending and asymmetric stretching of $\mathrm{PO}_{4}{ }^{3-}$ ions. To further verify the crystallinity of obtained AuNR@PAA/CaP yolk-shell NPs, the XRD pattern of the sample was carried out. The diffraction peaks well match the planes of $\mathrm{Au}$ crystals, indicating the formation of crystalline $\mathrm{Au}$. In addition, the characteristic peak at around $25^{\circ}-35^{\circ}$ is assigned to the amorphous $\mathrm{CaP}$ (Fig. S3, ESI). Surface information of the AuNR@PAA/CaP yolkshell NPs was characterized by XPS in Fig. S4. The peaks at 190 and $132 \mathrm{eV}$ are assigned to $\mathrm{P} 2 s$ and $\mathrm{P} 2 p$, respectively. The $\mathrm{Ca} 2 s$ and $\mathrm{Ca} 2 p$ peaks appear at binding energies of 438 and $347 \mathrm{eV}$, and the binding energies of 531 and $87 \mathrm{eV}$ are attributed to $\mathrm{O} 1 s$ and $\mathrm{Au} 4 f$, respectively. EDX spectrum confirms that the AuNR@PAA/CaP yolk-shell NPs are composed of $\mathrm{Au}, \mathrm{Ca}, \mathrm{C}, \mathrm{P}, \mathrm{O}$ (Fig. S5, ESI). Meanwhile, an ICP-AES analysis quantified the weight percentage of $\mathrm{Au}$ and $\mathrm{Ca}$ is $13.5 \%$ and $19.4 \%$, respectively.

\subsection{Photothermal Effect}

The AuNR@PAA/CaP yolk-shell NPs display a strong absorption peak at $806 \mathrm{~nm}$ (Fig. 1f), making it possible to be a photothermal treatment agent. To acquaint the photothermal performance, the yolk-shell NPs with different $\mathrm{Au}$ concentrations $(0,0.4,0.5,0.7$, and $0.9 \mathrm{mM}$ at $1.0 \mathrm{~W} \mathrm{~cm}^{-2}$ ) were irradiated with a NIR laser $(808 \mathrm{~nm})$ for $300 \mathrm{~s}$, and the temperature was monitored by a digital thermometer (Fig. 2a, b). Meanwhile, the concentration of $0.5 \mathrm{mM} \mathrm{Au}$ irradiated with various lasers power densities $\left(0.5,1.0,1.5\right.$, and $\left.2.0 \mathrm{~W} \mathrm{~cm}^{-2}\right)$ was also performed in Fig. 2c. It is obviously found that the temperature increasing depends on the concentration, lasers power density and irradiation duration. The Au concentrations of solutions greater than $0.5 \mathrm{mM}$ can be easily heated from $22{ }^{\circ} \mathrm{C}$ at least to $42{ }^{\circ} \mathrm{C}$. It is known that cancer cells can be effectively killed above $42^{\circ} \mathrm{C}$. As a negative control group, the temperature of water is stable at $22^{\circ} \mathrm{C}$. In addition, the photothermal conversion efficiency $(\eta)$ of the AuNR@PAA/CaP yolk-shell NPs was determined to be $26 \%$ (Fig. S6, ESI). As shown in Fig. 2d, no significant decrease of the temperature elevation is observed of the AuNR@PAA/CaP yolk-shell NPs after four repeated laser on-and-off cycles (the laser was on for about $5 \mathrm{~min}$ in each cycle) and the TEM image also indicates that the "rodlike" structure of AuNRs stay unchanged in the PAA/CaP shell (Fig. S7, ESI). Thus, our AuNR@PAA/CaP yolkshell NPs possess good photostability and constant photothermal conversion behavior.

Next, to investigate the photothermal effect in vitro, the fluorescence microscopy images were obtained by staining the live cells with calcein AM, which can emit strong green fluorescence in live cells. As shown in Fig. 2e, when the HeLa cells were treated with both 808-nm laser irradiation and AuNR@PAA/CaP yolk-shell NPs, the dark region matches the laser irradiation area very well, suggesting the death of HeLa cells upon exposure to the laser irradiation. As a contrast, the cell viability and cell density are not reduced when the samples were treated by only the yolkshell NPs or laser irradiation, compared with the control group. Obviously, the results illustrate that the yolk-shell NPs can transform laser energy into heat energy, which could kill HeLa cells and reduce adverse side effects to normal tissues as photothermal agents.

\subsection{DOX Loading, pH/NIR-Responsive Controlled Release and Cytotoxicity Assays in vitro}

To further evaluate the drug loading capacity, $\mathrm{pH} / \mathrm{NIR}$ controlled release behavior and cytotoxicity assays of the AuNR@PAA/CaP yolk-shell NPs, we selected DOX as a modal anticancer drug. The loading capacity of the AuNR@PAA/CaP yolk-shell NPs is evaluated by measuring the DOX concentration in solution before and after loading into the yolk-shell NPs using UV-Vis spectrophotometer measurements at $480 \mathrm{~nm}$ (Fig. 3a). The absorption intensity of DOX decreases significantly, indicating that the DOX has been stored in the yolk-shell NPs. The loading efficiency of DOX molecules into the AuNR@PAA/CaP yolk-shell NPs can reach up to approximately $100 \%$, and the loading content is $1 \mathrm{mg}$ of 
DOX per mg of the yolk-shell NPs. This ultrahigh loading capacity is major attributed to the void spaces, because of the thin $\mathrm{CaP}$ shell of about $10 \mathrm{~nm}$, which can load a small amount of the drug molecules. Figure $3 b$ shows different cumulative DOX-release profiles from the DOX-loaded AuNR@PAA/CaP yolk-shell NPs under different conditions at $37{ }^{\circ} \mathrm{C}$. In neutral phosphate buffered saline (PBS), which is similar to the normal tissue environment ( $\mathrm{pH} 7.4$ ), only $10 \%$ of DOX is released within $2 \mathrm{~h}$ and there is almost no release afterward (Fig. S8, ESI). Whereas the cumulative drug release amount attains $45.8 \%$ within the same period at mildly acidic environment $(\mathrm{pH} 5.0)$, which is similar to the extracellular $\mathrm{pH}$ of tumors, owing to the dissolution of $\mathrm{CaP}$ in acid environment. In order to further
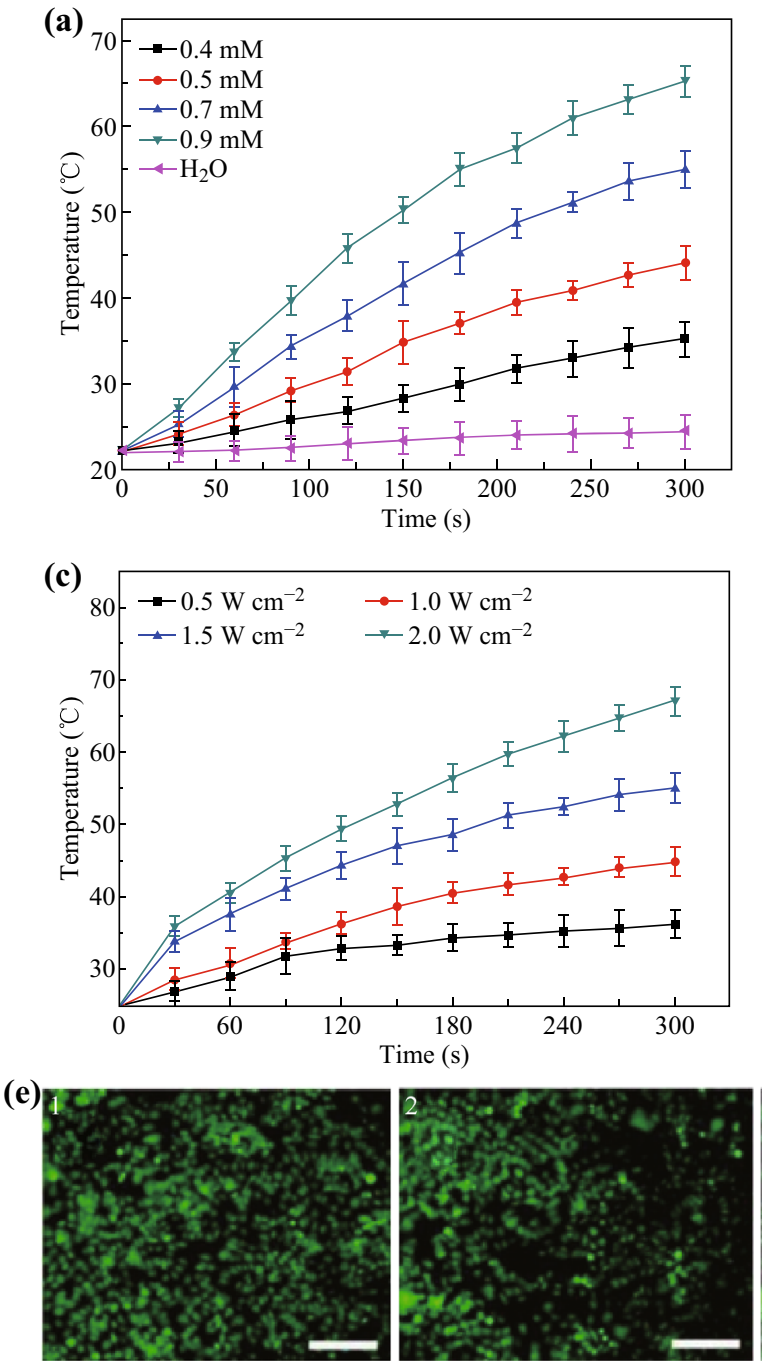

verify the dissolution of CaP NPs, we measured the content of $\mathrm{Ca}$ in the PBS (pH 5.0 and $\mathrm{pH} 7.4$ ) at different time points with ICP-AES (Fig. S9, ESI). The result shows that the content of $\mathrm{Ca}$ increases significantly with the extension of time ( $\mathrm{pH}$ 5.0), confirming that the $\mathrm{pH}$-responsive drug release of DOX results from the dissolution of $\mathrm{CaP}$, while the extremely slow release of $\mathrm{Ca}$ occurs in $\mathrm{pH}$ 7.4. The released $\mathrm{Ca}$ ions will not make much difference on cells, and owing to the yolk-shell NPs' high performance in photothermal effect and drug loading capacity, cancer cells can be ablated based on a small amount of DOX-loaded yolk-shell NPs $\left(50 \mu \mathrm{gL}^{-1}\right)$. Even the whole CaP shell is dissolved, and the $\mathrm{Ca}$ ions concentration is just $\sim 1.7 \times 10^{-7} \mathrm{M}$, which can still maintain a normal level

Fig. 2 a Variation of temperature as a function of different Au concentrations irradiated with an 808-nm laser (power density: $1.0 \mathrm{~W} \mathrm{~cm}^{-2}$ ), b Photothermal images of the yolk-shell NPs solution at different Au concentrations, and pure water exposed to the $808-\mathrm{nm}^{1} \mathrm{laser}\left(1.0 \mathrm{~W} \mathrm{~cm}^{-2}\right)$ recorded at different time intervals, c NIR-induced temperature increase at various lasers power densities in aqueous solution ( $808 \mathrm{~nm}, 0.5 \mathrm{mM})$, d Temperature monitoring of the yolk-shell NPs aqueous suspension (the Au concentration: $0.5 \mathrm{mM}$ ) during for successive cycles of an on-andoff laser, e Fluorescence microscopy images of HeLa cells with different treatments via staining with calcein AM: (1) control; (2) yolk-shell NPs only; (3) laser irradiation only; (4) yolk-shell NPs with laser irradiation (scale bars: $200 \mu \mathrm{m}$ ) 
[44]. In addition, we also investigated the promotional effect of NIR irradiation on DOX release during the experiment. In a certain time interval, the DOX-loaded yolk-shell NPs were exposed to an $808 \mathrm{~nm}$ NIR laser $(\mathrm{pH}$ 5.0). As shown in Fig. 3b, a burst release of DOX occurs upon applying the NIR laser. As a result of the photothermal effect of the yolk-shell NPs, the drug release upon NIR irradiation enhances significantly because the generated heat can effectively induce DOX release.

All the results demonstrate that the AuNR@PAA/CaP yolk-shell NPs can achieve pH/NIR dual-responsive release, making it possible for minimizing the drug stimulation to normal cells and improving antitumor efficacy. As depicted in Fig. 3c, the cell viability was as high as 98.2\% when the HeLa cells were treated with AuNR@PAA/CaP yolk-shell NPs at a high concentration of $50 \mu \mathrm{g} \mathrm{mL}^{-1}$, proving that the yolk-shell NPs exhibit excellent biocompatibility. DOX-loaded AuNR@PAA/ CaP yolk-shell NPs reveal a similar cell toxicity against HeLa cells with the same concentration of DOX, confirming that the yolk-shell NPs are effective drug vehicles. It should be noted that single yolk-shell NPs and DOX- loaded yolk-shell NPs under 808-nm laser irradiation, respectively, exhibit different ability for photothermal ablation of cancer cells, and the latter shows a higher cell toxicity. The results indicate that the combination of the chemo- and photothermal therapy of DOX-loaded AuNR@PAA/CaP yolk-shell NPs could significantly enhance the efficiency of tumor ablation.

\subsection{Confocal Laser Scanning Microscopy}

To further examine the time-dependent cell uptake and the intracellular release behavior of DOX-loaded AuNR@PAA/CaP yolk-shell NPs, the confocal laser scanning microscopy (CLSM) images of HeLa cells incubated with DOX-loaded AuNR@PAA/CaP yolk-shell NPs at different time points $(0.5,2$, and $6 \mathrm{~h})$ were collected. As shown in Fig. 4, the CLSM images were obtained for the red fluorescence of DOX and the blue fluorescence of Hoechst 33,342 after the nuclei was stained. With the increase in the incubation time, the DOX molecules gathered in the cell nucleus gradually increased, which suggests that DOXloaded NPs could facilitate cellular internalization and final (a)

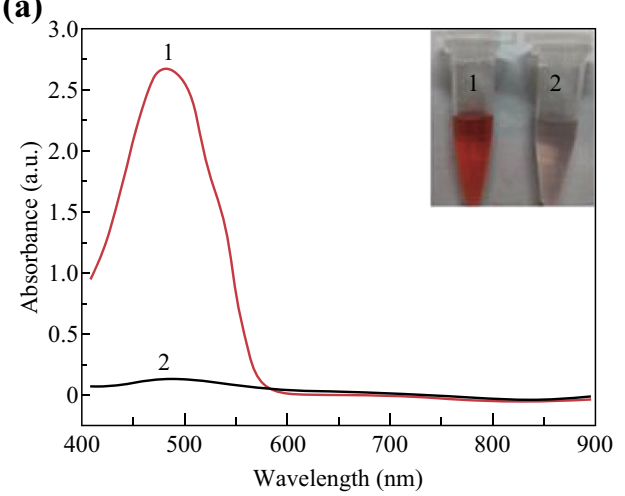

(b)

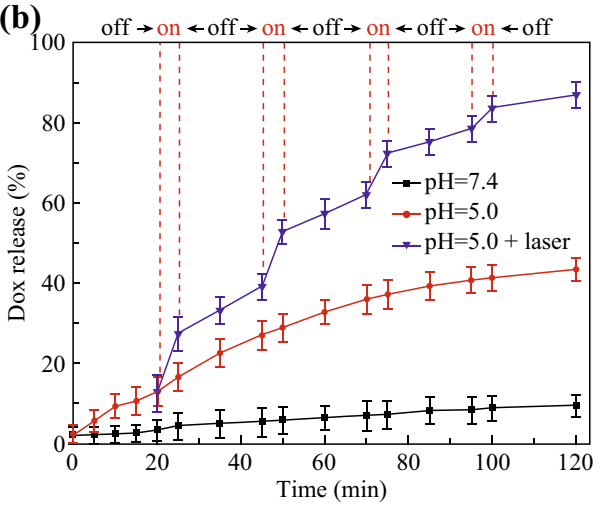

(c)

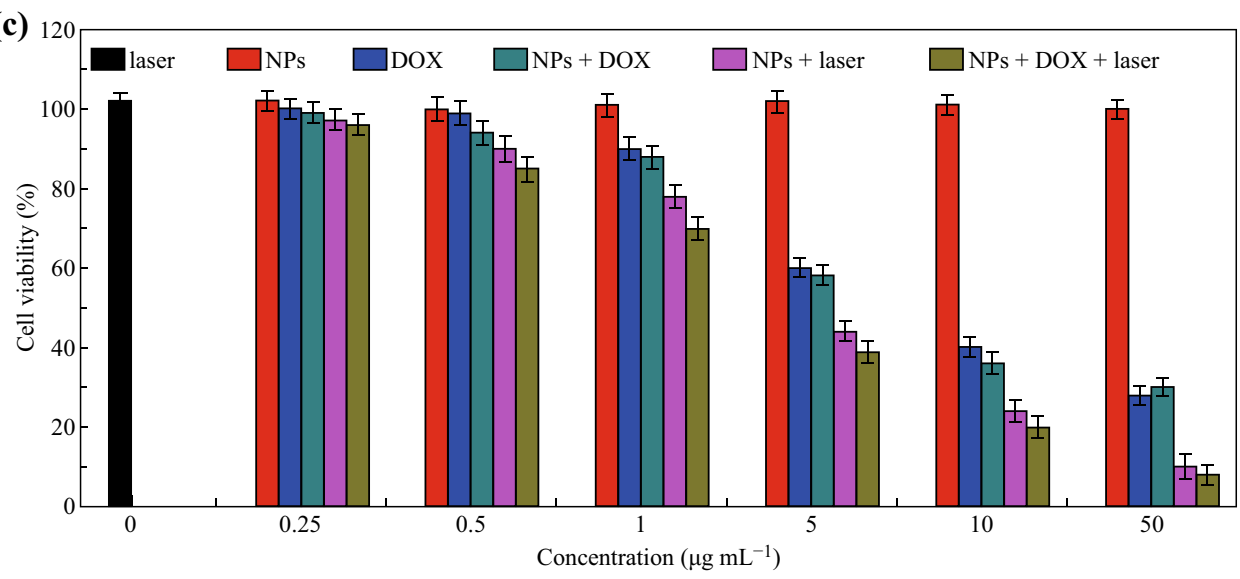

Fig. 3 a UV-Vis absorption spectra and photographs (inset) of original DOX aqueous solution (1) and the residual DOX content in the supernatant after interaction with AuNR@PAA/CaP yolk-shell NPs (2); b DOX-release profiles of DOX from DOX-loaded yolk-shell NPs in PBS buffer: $\mathrm{pH}=7.4,5.0$ and 5.0, with periodic laser ON/OFF irradiation $\left(808 \mathrm{~nm}, 1.0 \mathrm{~W} \mathrm{~cm}^{-2}\right)$, c Cytotoxicity assays of HeLa cells incubated with different treatments (laser, NPs, DOX, NPs + DOX, NPs + laser and NPs + DOX + laser, laser irradiation: $1.0 \mathrm{~W} \mathrm{~cm}^{-2}, 5 \mathrm{~min}$ ) 

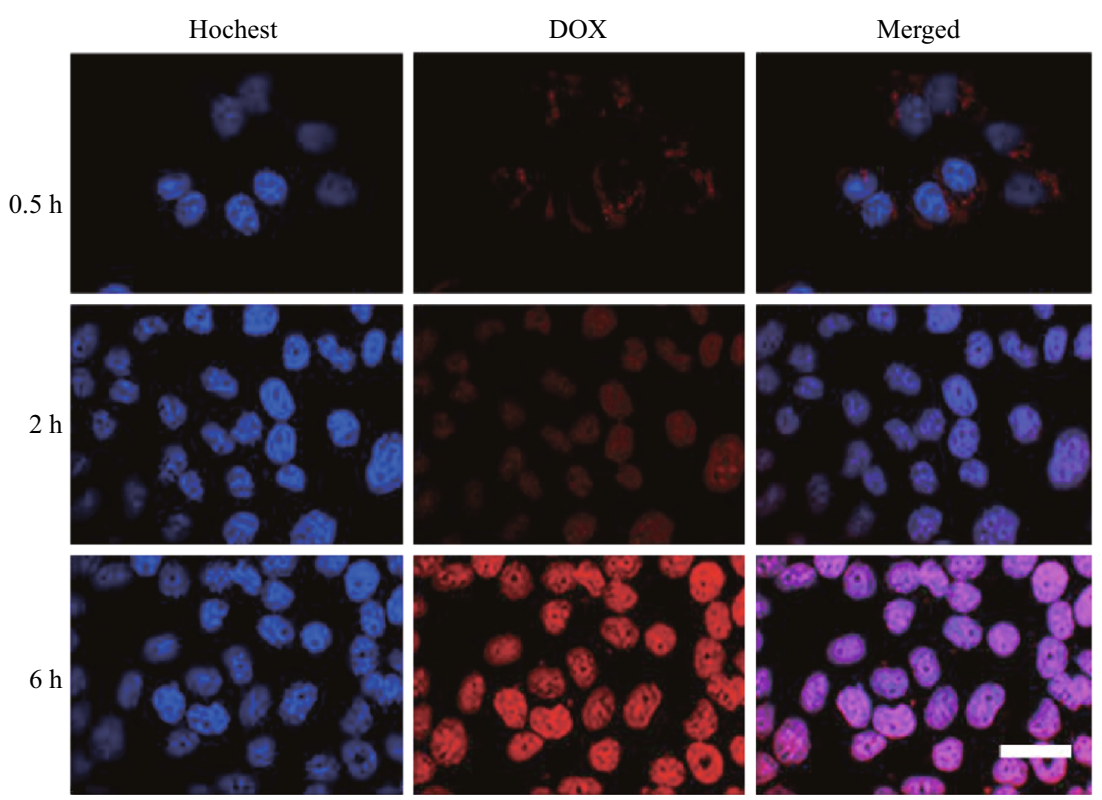

Fig. 4 CLSM images of HeLa cells incubated with DOX-loaded AuNR@PAA/CaP yolk-shell NPs for 0.5, 2, and 6 h (scale bar: 10 $\mu$ m)

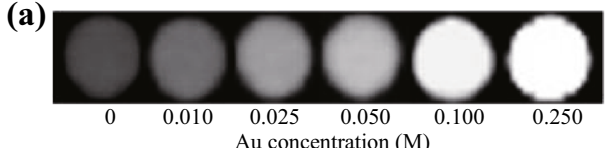

(b)

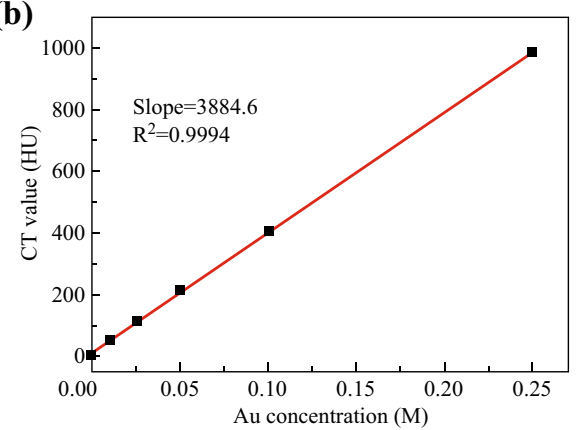

(c)

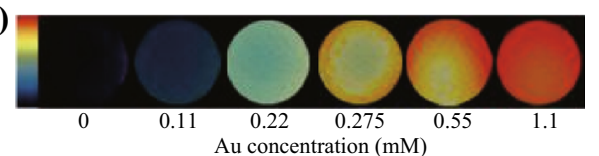

(d)

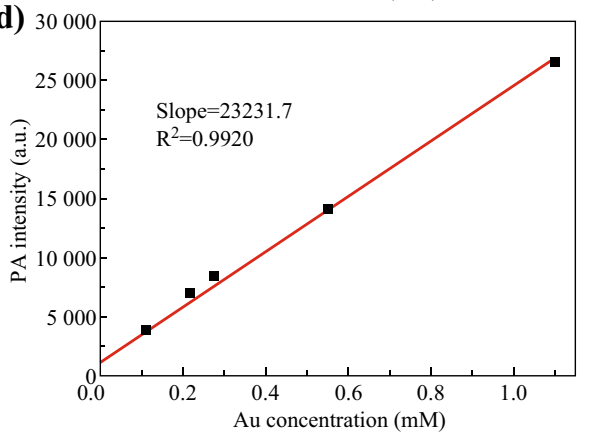

Fig. 5 a CT images of AuNR@PAA/CaP yolk-shell NPs and b linear relationship of CT signal intensities versus Au concentrations, $\mathbf{c}$ PA images and $\mathbf{d}$ linear relationship of PA signal intensities with different Au concentrations

delivery of DOX to the cell nuclei. We could conclude that the AuNR@PAA/CaP yolk-shell NPs have great potential as promising drug delivery nanocarriers to cancer cells.

\subsection{CT and PA Imaging in vitro}

The Au-based NPs have been considered as a promising $\mathrm{CT}$ imaging contrast agent, due to the presence of $\mathrm{Au}$ which has a higher X-ray absorption coefficient than iodine agents of conventional CT [45-47]. Therefore, we assessed the CT contrast efficacy of the AuNR@PAA/CaP yolkshell NPs. The CT signals enhance with increasing NPs concentrations (Fig. 5a). The HUs exhibit a well-correlated linear relationship in Fig. $5 \mathrm{~b}\left(R^{2}=0.9994\right)$. These results suggest that AuNR@PAA/CaP yolk-shell NPs are good candidates as positive $\mathrm{CT}$ imaging contrast agents. In addition, the high absorption coefficient of the AuNR@PAA/CaP yolk-shell NPs in the NIR region also makes them effective contrast agents for PA imaging. As shown in Fig. 5c, d, the PA signals of the NP solutions are enhanced with increasing NPs concentrations, displaying a concentration dependent manner. All the above results show that the AuNR@PAA/CaP yolk-shell NPs should be a promising candidate for dual-mode $\mathrm{CT} / \mathrm{PA}$ imaging. 


\section{Conclusions}

In summary, we have successfully fabricated novel AuNR@PAA/CaP yolk-shell NPs which possess ultrahigh anticancer drug loading, superior photothermal conversion ability, good biocompatibility and pH/NIR dual-responsiveness. The yolk-shell NPs can serve as promising theranostic agents for simultaneous dual-mode CT/PA imaging and chemo-photothermal therapy. Furthermore, this work could encourage further study in the construction of CaP-based multifunctional yolk-shell NPs using NIR absorbing, fluorescent and magnetic nanomaterials for cancer theranostics.

Acknowledgements We would like to thank the National Natural Science Foundation of China (Grant Nos. 21573040 and 21603029), the Natural Science Foundation and Science and Technology Development Planning of Jilin Province (20150204086GX and 20170520148JH), the Fundamental Research Funds for the Central Universities (2412016KJ007 and 2412016KJ020), the China Postdoctoral Science Foundation (2016M600224), the Jilin Provincial Research Foundation for Basic Research (20160519012JH) and Jilin Provincial Key Laboratory of Advanced Energy Materials (Northeast Normal University).

Open Access This article is distributed under the terms of the Creative Commons Attribution 4.0 International License (http://crea tivecommons.org/licenses/by/4.0/), which permits unrestricted use, distribution, and reproduction in any medium, provided you give appropriate credit to the original author(s) and the source, provide a link to the Creative Commons license, and indicate if changes were made.

\section{References}

1. Q. Chen, Z. Liu, Albumin carriers for cancer theranostics: a conventional platform with new promise. Adv. Mater. 28(47), 10557-10566 (2016). doi:10.1002/adma.201600038

2. L. Li, L.Y. Zhang, T.T. Wang, X.T. Wu, H. Ren, C.G. Wang, Z.M. Su, Facile and scalable synthesis of novel spherical Au nanocluster assemblies@polyacrylic acid/calcium phosphate nanoparticles for dual-modal imaging-guided cancer chemotherapy. Small 11(26), 3162-3173 (2015). doi:10.1002/smll. 201403517

3. Z.J. Zhang, J. Wang, C.Y. Chen, Near-infrared light-mediated nanoplatforms for cancer thermo-chemotherapy and optical imaging. Adv. Mater. 25(28), 3869-3880 (2013). doi:10.1002/ adma.201301890

4. X. Ding, J.H. Liu, J.Q. Li, F. Wang, Y.H. Wang, S.Y. Song, H.J. Zhang, Polydopamine coated manganese oxide nanoparticles with ultrahigh relaxivity as nanotheranostic agents for magnetic resonance imaging guided synergetic chemo-/photothermal therapy. Chem. Sci. 7(11), 6695-6700 (2016). doi:10.1039/ C6SC01320A

5. S. Panesar, S. Neethirajan, Microfluidics: rapid diagnosis for breast cancer. Nano-Micro Lett. 8(3), 204-220 (2016). doi:10. 1002/adfm.201403885

6. A.B. Reddy, B. Manjula, T. Jayaramudu, E.R. Sadiku, P.A. Babu, S.P. Selvam, 5-Fluorouracil loaded chitosan-PVA/Na + MMT nanocomposite films for drug release and antimicrobial activity. Nano-Micro Lett. 8(3), 260-269 (2016). doi:10.1007/s40820016-0086-410

7. J.S. Lee, W.L. Murphy, Functionalizing calcium phosphate biomaterials with antibacterial silver particles. Adv. Mater. 25(8), 1173-1179 (2013). doi:10.1002/adma.201203370

8. X. Li, X.P. Wang, Y. Sogo, T. Ohno, K. Onuma, A. Ito, Mesoporous silica-calcium phosphate-tuberculin purified protein derivative composites as an effective adjuvant for cancer immunotherapy. Adv. Healthc. Mater. 2(6), 863-871 (2013). doi:10.1002/adhm.201200149

9. P. Shi, K.G. Qu, J.S. Wang, M. Li, J.S. Ren, X.G. Qu, pHresponsive NIR enhanced drug release from gold nanocages possesses high potency against cancer cells. Chem. Commun. 48(61), 7640-7642 (2012). doi:10.1039/c2cc33543c

10. C.Y. Wang, L.Y. Zhang, S.N. Li, M.J. Zhang, T.T. Wang, L. Li, C.G. Wang, Z.M. Su, A designed synthesis of multifunctional $\mathrm{Fe}_{3} \mathrm{O}_{4} @$ carbon/zinc phosphate nanoparticles for simultaneous imaging and synergic chemo-photothermal cancer therapy. J. Mater. Chem. B 4(35), 5809-5813 (2016). doi:10.1039/ C6TB01669C

11. M. Nakamura, A. Oyane, Physicochemical fabrication of calcium phosphate-based thin layers and nanospheres using laser processing in solutions. J. Mater. Chem. B 4(38), 6289-6301 (2016). doi:10.1039/C6TB01362G

12. W.M. Li, S.Y. Chen, D.M. Liu, In situ doxorubicin-CaP shell formation on amphiphilic gelatin-iron oxide core as a multifunctional drug delivery system with improved cytocompatibility, pH-responsive drug release and MR imaging. Acta Biomater. 9(2), 5360-5368 (2013). doi:10.1016/j.actbio.2012.09.023

13. X.J. Chen, M.J. Zhang, S.N. Li, L. Li, L.Y. Zhang, T.T. Wang, M. Yu, Z.C. Mou, C.G. Wang, Facile synthesis of polypyrrole@metal-organic framework core-shell nanocomposites for dual-mode imaging and synergistic chemo-photothermal therapy of cancer cells. J. Mater. Chem. B 5, 1772-1778 (2017). doi:10.1039/C6TB03218D

14. M.M. Sun, F. Liu, Y.K. Zhu, W.S. Wang, J. Hu et al., Saltinduced aggregation of gold nanoparticles for photoacoustic imaging and photothermal therapy of cancer. Nanoscale 8(8), 4452-4457 (2016). doi:10.1039/C6NR00056H

15. P. Huang, P.F. Rong, J. Lin, W.W. Li, X.F. Yan et al., Triphase interface synthesis of plasmonic gold bellflowers as near-infrared light mediated acoustic and thermal theranostics. J. Am. Chem. Soc. 136(23), 8307-8313 (2014). doi:10.1021/ja503115n

16. A. Li, X. Li, X.J. Yu, W. Li, R.Y. Zhao, X. An, D.X. Cui, X.Y. Chen, W.W. Li, Synergistic thermoradiotherapy based on PEGylated $\mathrm{Cu}_{3} \mathrm{BiS}_{3}$ ternary semiconductor nanorods with strong absorption in the second near-infrared window. Biomaterials 112, 164-175 (2017). doi:10.1016/j.biomaterials.2016.10.024

17. L. Guo, G.L. Niu, X.L. Zheng, J.C. Ge, W.M. Liu, Q.Y. Jia, P.P. Zhang, H.Y. Zhang, P.F. Wang, Single near-infrared emissive polymer nanoparticles as versatile phototheranostics. Adv. Sci. (2017). doi:10.1002/advs.201700085

18. S.Y. Shi, Y.J. Liu, Y. Chen, Z.H. Zhang, Y.S. Ding, Z.Q. Wu, J. Yin, L.M. Nie, Versatile pH-response micelles with high cellpenetrating helical diblock copolymers for photoacoustic imaging guided synergistic chemo-photothermal therapy. Theranostics 6(12), 2170-2182 (2016). doi:10.7150/thno.16633

19. Y.Y. Li, C.H. Jiang, D.W. Zhang, Y. Wang, X.Y. Ren, K.L. Ai, X.S. Chen, L.H. Ru, Targeted polydopamine nanoparticles enable photoacoustic imaging guided chemo-photothermal synergistic therapy of tumor. Acta Biomater. 47, 124-134 (2017). doi:10. 1016/j.actbio.2016.10.010

20. W.W. Li, P.F. Rong, K. Yang, P. Huang, K. Sun, X.Y. Chen, Semimetal nanomaterials of antimony as highly efficient agent 
for photoacoustic imaging and photothermal therapy. Biomaterials 45, 18-26 (2015). doi:10.1016/j.biomaterials.2014.12.037

21. J. Liu, P.Y. Wang, X. Zhang, L.M. Wang, D.L. Wang et al., Rapid degradation and high renal clearance of $\mathrm{Cu}_{3} \mathrm{BiS}_{3}$ nanodots for efficient cancer diagnosis and photothermal therapy in vivo. ACS Nano 10(4), 4587-4598 (2016). doi:10.1021/acsnano. $6 \mathrm{~b} 00745$

22. S. Duan, Y.J. Yang, C.L. Zhang, N.N. Zhao, F.J. Xu, NIR-responsive polycationic gatekeeper-cloaked hetero-nanoparticles for multimodal imaging-guided triple-combination therapy of cancer. Small 13(9), 1603133-1603142 (2017). doi:10.1002/smll. 201603133

23. J.B. Qin, Z.Y. Peng, B. Li, K.C. Ye, Y.X. Zhang et al., Gold nanorods as a theranostic platform for in vitro and in vivo imaging and photothermal therapy of inflammatory macrophages. $\begin{array}{llll}\text { Nanoscale 7(33), 13991-14001 (2015). doi:10.1039/ } & \end{array}$ C5NR02521D

24. F. Gao, L.Y. Bai, S.Y. Liu, R.C. Zhang, J.T. Zhang, X.H. Feng, Y.J. Zheng, Y.L. Zhao, Rationally encapsulated gold nanorods improving both linear and nonlinear photoacoustic imaging contrast in vivo. Nanoscale 9, 79-86 (2017). doi:10.1039/ C6NR07528B

25. J.B. Song, X.Y. Yang, O. Jacobson, P. Huang, X.L. Sun et al., Ultrasmall gold nanorod vesicles with enhanced tumor accumulation and fast excretion from the body for cancer therapy. Adv. Mater. 27(33), 4910-4917 (2015). doi:10.1002/adma.201502486

26. G.F. Luo, W.H. Chen, Q. Lei, W.X. Qiu, Y.X. Liu, Y.J. Cheng, X.Z. Zhang, A triple-collaborative strategy for high-performance tumor therapy by multifunctional mesoporous silica-coated gold nanorods. Adv. Funct. Mater. 26(24), 4339-4350 (2016). doi:10. 1002/adfm.201505175

27. A. Khanal, C. Ullum, C.W. Kimbrough, N.C. Garbett, J.A. Burlison et al., Tumor targeted mesoporous silica-coated gold nanorods facilitate detection of pancreatic tumors using multispectral optoacoustic tomography. Nano Res. 8(12), 3864-3877 (2015). doi:10.1007/s12274-015-0886-8

28. J.V. Jokerst, A.J. Cole, D.S.D. Van, S.S. Gambhir, Gold nanorods for ovarian cancer detection with photoacoustic imaging and resection guidance via Raman imaging in living mice. ACS Nano 6(11), 10366-10377 (2012). doi:10.1021/nn304347g

29. P. Huang, L. Bao, C. Zhang, J. Lin, T. Luo et al., Folic acidconjugated silica-modified gold nanorods for X-ray/CT imagingguided dual-mode radiation and photo-thermal therapy. Biomaterials 32(36), 9796-9809 (2011). doi:10.1016/j.biomaterials. 2011.08.086

30. J. Wu, Y. Liu, Y.X. Tang, S.J. Wang, C.Y. Wang et al., Synergistic chemo-photothermal therapy of breast cancer by mesenchymal stem cell-encapsulated yolk-shell GNR@HPMO-PTX nanospheres. ACS Appl. Mater. Interfaces. 8(28), 17927-17935 (2016). doi:10.1021/acsami.6b05677

31. Y.F. Zhu, J.L. Shi, W.H. Shen, X.P. Dong, J.W. Feng, M.L. Ruan, Y.S. Li, Stimuli-responsive controlled drug release from a hollow mesoporous silica sphere/polyelectrolyte multilayer core-shell structure. Angew. Chem. Int. Ed. 44(42), 5083-5087 (2015). doi:10.1002/anie.200501500

32. J. Gao, G. Liang, B. Zhang, Y. Kuang, X. Zhang, B. Xu, FePt@ $\mathrm{CoS}_{2}$ yolk-shell nanocrystals as a potent agent to kill HeLa cells. J. Am. Chem. Soc. 129(5), 1428-1433 (2007). doi:10. 1021/ja067785e

33. J. Kim, H.S. Kim, N. Lee, T. Kim, H. Kim, T. Yu, I.C. Song, W.K. Moon, T. Hyeon, Multifunctional uniform nanoparticles composed of a magnetite nanocrystal core and a mesoporous silica shell for magnetic resonance and fluorescence imaging and for drug delivery. Angew. Chem. Int. Ed. 47(44), 8438-8441 (2008). doi:10.1002/anie.200802469

34. H. Wu, G. Liu, S. Zhang, J. Shi, L. Zhang, Y. Chen, F. Chen, H. Chen, Biocompatibility, MR imaging and targeted drug delivery of a rattle-type magnetic mesoporous silica nanosphere system conjugated with PEG and cancer-cell-specific ligands. J. Mater. Chem. 21(9), 3037-3045 (2011). doi:10.1039/c0jm02863k

35. J. Liu, S.Z. Qiao, S.B. Hartono, G.Q. Lu, Monodisperse yolkshell nanoparticles with a hierarchical porous structure for delivery vehicles and nanoreactors. Angew. Chem. Int. Ed. 49(29), 4981-4985 (2010). doi:10.1002/anie.201001252

36. S.X. Li, J.Z. Zheng, D.J. Chen, Y.J. Wu, W.X. Zhang, F.Y. Zheng, J. Cao, H.R. Ma, Y.L. Liu, Yolk-shell hybrid nanoparticles with magnetic and $\mathrm{pH}$-sensitive properties for controlled anticancer drug delivery. Nanoscale 5(23), 11718-11724 (2013). doi:10.1039/c3nr04032a

37. L. Zhao, J. Peng, Q. Huang, C. Li, M. Chen, Y. Sun, Q. Lin, L. Zhu, F. Li, Near-infrared photoregulated drug release in living tumor tissue via yolk-shell upconversion nanocages. Adv. Funct. Mater. 24(3), 363-371 (2014). doi:10.1002/adfm.201302133

38. H. Wu, G.Y. Zheng, N. Liu, T.J. Carney, Y. Yang, Y. Cui, Engineering empty space between Si nanoparticles for lithiumion battery anodes. Nano Lett. 12(2), 904-909 (2012). doi:10. $1021 / \mathrm{nl} 203967 \mathrm{r}$

39. C. Guan, X.H. Wang, Q. Zhang, Z.X. Fan, H. Zhang, H.J. Fan, Highly stable and reversible lithium storage in $\mathrm{SnO}_{2}$ nanowires surface coated with a uniform hollow shell by atomic layer deposition. Nano Lett. 14(8), 4852-4858 (2014). doi:10.1021/ nl502192p

40. Z.Y. Cai, L. Xu, M.Y. Yan, C.H. Han, L. He et al., Manganese oxide/carbon yolk-shell nanorod anodes for high capacity lithium batteries. Nano Lett. 15(1), 738-744 (2015). doi:10.1021/ n1504427d

41. Q.Y. Li, F. Wang, L.Q. Sun, Z. Jiang, T. Ye et al., Design and synthesis of $\mathrm{Cu} @ \mathrm{CuS}$ yolk-shell structures with enhanced photocatalytic activity. Nano-Micro Lett. 9, 35 (2017). doi:10.1007/ s40820-017-0135-7

42. X.J. Yang, X. Liu, Z. Liu, F. Pu, J.S. Ren, X.G. Qu, Near-infrared light-triggered, targeted drug delivery to cancer cells by aptamer gated nanovehicles. Adv. Mater. 24(21), 2890-2895 (2012). doi:10.1002/adma.201104797

43. J. Zuloaga, E. Prodan, P. Nordlander, Quantum plasmonics: optical properties and tunability of metallic nanorods. ACS Nano 4(9), 5269-5276 (2010). doi:10.1021/nn101589n

44. G.A. Morrill, H.R. Kaback, E. Robbins, Effect of calcium on intracellular sodium and potassium concentrations in plant and animal cells. Nature 204(4959), 641-642 (1964). doi:10.1038/ $204641 \mathrm{a} 0$

45. J. Michaelis, C. Hettich, J. Mlynek, V. Sandoghdar, Optical microscopy using a single-molecule light source. Nature 405(6784), 325-328 (2000). doi:10.1038/35012545

46. T. Kim, N. Lee, D.R. Arifin, I. Shats, M. Janowski, P. Walczak, T. Hyeon, J.W.M. Bulte, In vivo micro-CT imaging of human mesenchymal stem cells labeled with gold-poly-L-Lysine nanocomplexes. Adv. Funct. Mater. 27(3), 1604213-1604220 (2016). doi:10.1002/adfm.201604213

47. X. Li, C. Wang, H. Tan, L.L. Cheng, G.B. Liu et al., Gold nanoparticles-based SPECT/CT imaging probe targeting for vulnerable atherosclerosis plaques original research article. Biomaterials 108, 71-80 (2016). doi:10.1016/j.biomaterials.2016.08. 048 